Article

\title{
Effects of Oxalic Acid on Apis mellifera (Hymenoptera: Apidae)
}

\author{
Eva Rademacher*, Marika Harz and Saskia Schneider \\ Institute of Biology/Neurobiology, Freie Universität Berlin, Königin-Luise-Str. 28-30, 14195 Berlin, Germany; \\ marika.harz@lwk.nrw.de (M.H.); saskia.schneider@kabelmail.de (S.S.) \\ * Correspondence: e.rademacher@fu-berlin.de; Tel.: +49-30-8385-6537
}

Academic Editor: Brian T. Forschler

Received: 12 June 2017; Accepted: 2 August 2017; Published: 7 August 2017

\begin{abstract}
Oxalic acid dihydrate is used to treat varroosis of Apis mellifera. This study investigates lethal and sublethal effects of oxalic acid dihydrate on individually treated honeybees kept in cages under laboratory conditions as well as the distribution in the colony. After oral application, bee mortality occurred at relatively low concentrations (No Observed Adverse Effect Level (NOAEL) $50 \mu \mathrm{g} / \mathrm{bee}$; Lowest Observed Adverse Effect Level (LOAEL) $75 \mu \mathrm{g} / \mathrm{bee}$ ) compared to the dermal treatment (NOAEL $212.5 \mu \mathrm{g} / \mathrm{bee}$; LOAEL $250 \mu \mathrm{g} / \mathrm{bee}$ ). The dosage used in regular treatment via dermal application (circa $175 \mu \mathrm{g} /$ bee) is below the LOAEL, referring to mortality derived in the laboratory. However, the treatment with oxalic acid dihydrate caused sublethal effects: This could be demonstrated in an increased responsiveness to water, decreased longevity and a reduction in $\mathrm{pH}$-values in the digestive system and the hemolymph. The shift towards stronger acidity after treatment confirms that damage to the epithelial tissue and organs is likely to be caused by hyperacidity. The distribution of oxalic acid dihydrate within a colony was shown by macro-computed tomography; it was rapid and consistent. The increased density of the individual bee was continuous for at least 14 days after the treatment indicating the presence of oxalic acid dihydrate in the hive even long after a treatment.
\end{abstract}

Keywords: Apis mellifera; Varroa destructor; oxalic acid dihydrate; toxicity; tolerance; sublethal effects

\section{Introduction}

Oxalic acid dihydrate (OAD) is one of the most important organic acids used for the control of Varroa destructor. It has been known to be effective against the parasite since the end of the 20th century [1]. The European Group for Integrated Varroa Control developed OAD for the final application stage in beekeeping [2,3]. Three different application methods of OAD exist: trickling, spraying and evaporation. There are principal points to be considered concerning the medical treatment of honey bee colonies: the tolerability of the ingredient to bees, and the toxicity to mites, as well as its distribution in the colony, which again is directly affects the toxicity and efficacy of a substance. The trickling method of OAD combines high efficacy against $V$. destructor and low bee mortality. The tolerability in the bee colony has been documented in a concentration of 3.5\% (w/v) OAD and a dose of 30-50 mL per colony for Central Europe [4]. Approval as a veterinary drug has been given in many countries worldwide over recent years, for Germany in 2006 [5].

So far, toxicological data on individual bees in the laboratory without combinatory effects has not been available. The mode of action in the colony was only partially clarified. In order to get a better understanding we tested the toxicity of OAD after dermal or oral application in the laboratory. Our aim was to define the no observed adverse effect level (NOAEL) and the lowest observed adverse effect levels (LOAEL) for OAD, including a safety margin for the dosage used in practical beekeeping. With this focus in mind, it is most important to look primarily at the dosage range of up to $10 \%$ bee 
mortality, as higher mortality rates are not acceptable for practical beekeeping. Therefore, we decided not to test very high dosages as it is unreasonable to kill large numbers of bees for non-relevant information. Furthermore, we wanted to understand which sublethal effects can be found and how $\mathrm{OAD}$ is distributed in the colony.

\section{Materials and Methods}

\subsection{Laboratory Tests: Treatment of Individual Bees with $O A D$}

\subsubsection{Investigation of Lethal Effects-Acute Oral and Dermal Toxicity}

The toxicity tests were conducted during August and September using Apis mellifera carnica bees from our apiary at the Institute of Biology/Neurobiology, Berlin (Germany). The colonies were managed according to good beekeeping practice.

Worker bees were recruited from brood combs by brushing about 100 individuals into small cages. The test bees were approximately five to ten days old. The cages are made of wood (105/65/120 mm length/width/height) with bee wire and a glass plate on the sides. The caged bees were kept under laboratory conditions in the dark at $22{ }^{\circ} \mathrm{C}$ and $65 \%$ relative humidity, draft-free. The bees formed small clusters and were starved for $24 \mathrm{~h}$ to ensure an even distribution of food before being treated with OAD. After the application, they were held in small groups of 10 bees per cage and received food (Apifonda, Südzucker AG, Mannheim, Germany) and water ad libitum.

OAD (Caelo, Hilden, Germany) dissolved in sucrose solution (50\% $w / w)$ was applied to bees individually using two application forms: trickling $5 \mu \mathrm{L}$ OAD solution onto the abdomen (dermal) or feeding $10 \mu \mathrm{L}$ OAD solution (oral). Topical treatment with $5 \mu \mathrm{L}$ was the maximum volume of solution applicable without considerable loss of agent. In feeding trials, $10 \mu \mathrm{L}$ solution allows the feeding of even high dosages by lower concentrations, so they were well accepted by the bees. The test design for both treatments was identical. Each dosage was tested on 30 bees (three cages per dosage and ten bees in every cage). Each trial was replicated at least once leading to a minimum number of 60 bees tested for each dosage and treatment method. The control groups (three cages with ten bees per cage, one replicate) were treated in the same way but received only sucrose solution $(50 \% w / w)$. The acute dermal toxicity test was conducted with different OAD concentrations: 3.5, 4.25, 5, 7.5 and $10 \%(w / v)$ and dosages: $175,212.5,250,375$ and $500 \mu \mathrm{g}$ OAD/bee, respectively. The acute oral toxicity test consisted of concentrations respectively dosages: $0.1,0.5,0.75,0.8$ and $1 \%(w / v)$ corresponding to $10,50,75,80$ and $100 \mu \mathrm{g}$ OAD/bee. Bee mortality data for test and control groups were collected 24, 48 and $72 \mathrm{~h}$ after the respective applications. Directly after the treatment, bees were observed for four hours and in time intervals up to $72 \mathrm{~h}$ for signs of behavioral changes.

\subsubsection{Investigation of Sublethal Effects}

Responsiveness to Water and Ascending Concentrations of Sucrose Solution

The proboscis extension response (PER) was used to test bees' responsiveness to water and ascending concentrations of sucrose solution (ACSS). Bees were recruited out of the colony as described for the previous experiments and individually marked. Bees were treated dermally with $5 \mu \mathrm{L}$ of $3.5 \%$ OAD in sucrose solution $(50 \% w / w)$ trickled onto the abdomen, the controls received sucrose solution only $(n=60)$. The proportion of animals releasing a PER was calculated for water and six concentrations of sucrose solution $(0.1 \%, 0.3 \%, 1 \%, 3 \%, 10 \%$ and $30 \%)$. Each bee was tested twice: prior to the treatment and $24 \mathrm{~h}$ afterwards. Between tests the bees were kept in cages and starved for three hours prior to each test to ensure equal motivation. Solutions were applied to the antennae with a three-minute inter-trial interval. To ensure equal motivation, bees were starved for $3 \mathrm{~h}$ prior to the tests. Without anesthetization, every bee was carefully moved into a little plastic tube allowing only the head with antennae and proboscis to move freely. Bees were tested for water and then for sucrose responsiveness 
by applying first a drop of water followed by the ascending sucrose solutions to the antennae with a 3-min inter-trial interval. The proportion of animals releasing a PER was calculated.

Longevity under Laboratory Conditions

To obtain bees of the same age, a brood-frame was taken from a colony and put into an incubator at $34.5^{\circ} \mathrm{C}$. The hatched bees were removed daily. The young bees were kept in small cages and provided with food (Apifonda, Südzucker AG, Mannheim, Germany) and water ad libitum. Pretests showed a mortality rate of up to $60 \%$ when bees younger than five days were treated with OAD; for this experiment bees at the age of five days were chosen and treated dermally with $5 \mu \mathrm{L}$ of $3.5 \%$ OAD in sucrose solution $(50 \% w / w)$ trickled onto the abdomen or sucrose solution (controls), respectively (n $=50$ ). Dead bees were counted and removed from the cages daily until the last bee had died. The test was repeated four times, a total of 200 bees per group were treated.

$\mathrm{pH}$ Values of the Digestive System and the Hemolymph

Worker bees were brushed from honey combs and individually treated with OAD. Dermal treatment was conducted with an amount of $5 \mu \mathrm{L} \mathrm{3.5 \%} \mathrm{OAD} \mathrm{in} \mathrm{sucrose} \mathrm{solution} \mathrm{(50 \%} w / w)$, dosage: $175 \mu \mathrm{g}$ /bee, $\mathrm{n}=120)$; oral treatment was performed with $10 \mu \mathrm{L} 0.35 \%$ OAD in sucrose solution (50\% $w / w$, dosage: $35 \mu \mathrm{g} /$ bee, $\mathrm{n}=120$ ). The test animals were kept in cages as described above. In intervals of 24,48 and $72 \mathrm{~h}$ post treatment the bees were frozen and subsequently dissected according to standard methods [6], the intestinal parts (crop, ventriculus and rectum) being removed and transferred onto micro-slides for $\mathrm{pH}$ measurement. An Inlab ${ }^{\circledR}$ Surface Electrode, which enables the $\mathrm{pH}$ measurement of very small amounts of liquid (minimum of $5 \mu \mathrm{L}$ ), was carefully placed onto the different parts of the digestive system. The electrode was connected to a FiveGo ${ }^{\mathrm{TM}} \mathrm{pH}$ meter (limits of error: $\pm 0.01 \mathrm{pH}$ ). The sampling of the hemolymph was conducted by the removal of the front and hind wings from the bee's thorax. A slight pressure on the thorax enabled the extraction of the hemolymph from the wing base. The droplet of the hemolymph was absorbed with micro-capillaries and immediately transferred to micro-slides for $\mathrm{pH}$ measurement. Hemolymph samples (minimum of five bees per sample) were pooled to gain at least $5 \mu \mathrm{L}$ of liquid.

\subsection{Computer Tomography of Honey Bee Colonies: Distribution of OAD}

Internal structures of a bee hive can be demonstrated by computed tomography [7]. Two honey bee nucleus colonies (A. m. carnica) were used for a distribution test with a macro-computed tomography scanner (macroCT). The colonies consisted of approximately 4000 individuals and had already formed a winter cluster. The treatments were conducted in November without brood. OAD $(3.5 \% w / v$ in sucrose solution $50 \% w / w$ ) was applied in the recommended dosage (according to package instructions for use-Oxuvar ${ }^{\circledR}$ ) by trickling onto the bees in the bee space. OAD was mixed with the water-soluble contrast agent Unilux ${ }^{\circledR}$ (Iopamidol, $370 \mathrm{mg}$ iodine $/ \mathrm{mL}$ ) in a dosage of $185 \mu \mathrm{g} / \mathrm{bee}$. The contrast agent Unilux showed no bee toxicity in a previous study [8].

For in-hive visualization of OAD distribution a macroCT scanner (Xvision, Toshiba) was used (Figure 1). With a helical scanner, a distance of $250 \mathrm{~mm}$ was examined ensuring the colony was captured completely. The CT images were reconstructed with a slice thickness of $2 \mathrm{~mm}$ (Table 1). For visualization via 2D images and data analysis we used the software eFilmTM LiteTM (MergeTM Healthcare 2008). The 2D images allowed the measuring of the density of individual bees in Hounsfield units (HU). This density is directly related to the amount of solution applied to the surface of the bee's bodies. In a defined area of $100 \mathrm{~cm}^{2}$ in the central area of the comb, as well as in the boundary area of the bee cluster, the density of single bees $(n=144)$ was measured over three combs. In one colony, the measurements were conducted before application (control) and $10 \mathrm{~min}$ respectively thirty minutes after applying OAD. The second colony measurements were conducted before application (control) and 3, 7 and 14 days respectively after applying OAD ( $\leq 211$ /group). Only bees placed parallel to the macroCT sectional plane were quantified. The total dosage of radiation for the scan of the entire 
bee colony was $249.8 \mathrm{mGy}$, spread over the helix distance of $250 \mathrm{~mm}$ (125 slices of $2 \mathrm{~mm}$ thickness). Compared to the dosage of $500 \mathrm{mGy}$ reported by [9] for biological effects in Drosophila melanogaster, this dosage can be considered harmless to bees.

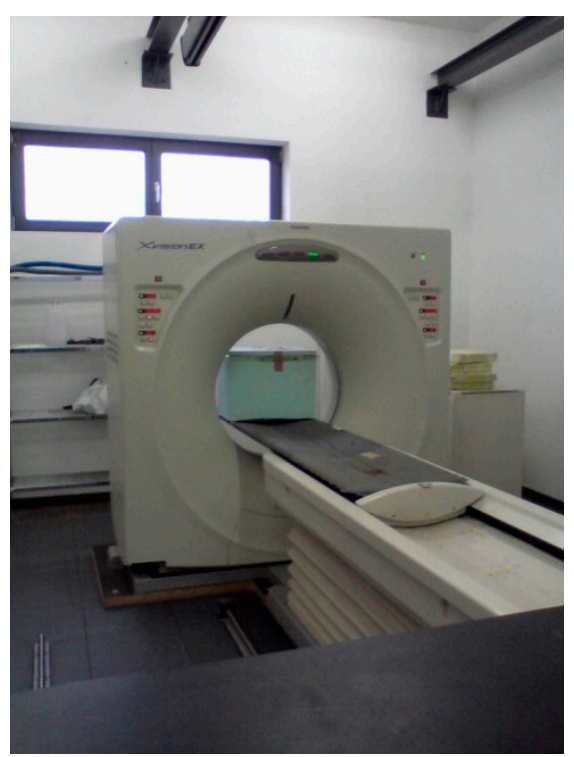

Figure 1. Bee colony placed in a macro-computed tomography scanner (macroCT).

Table 1. Technical data for A. mellifera colony scanning.

\begin{tabular}{cc}
\hline Parameter & Resolution \\
\hline Slice thickness & $2 \mathrm{~mm}$ \\
Pitch & 2.5 \\
Helix & $250 \mathrm{~mm}$ \\
Peak X ray voltage & $120 \mathrm{kV}$ \\
X ray tube current & $80 \mathrm{~mA}$ \\
Total scan time & $75 \mathrm{~s}$ \\
Matrix & $512 \times 512$ \\
Scan field of view & large \\
Display field of view & $480 \mathrm{~mm}$ \\
Window width & 1000 \\
Window level & -300 \\
Total scan dosage & $249.8 \mathrm{mGy}$ \\
\hline
\end{tabular}

\subsection{Statistical Analysis}

The statistical analysis was conducted using SigmaStat ${ }^{\circledR} 3.0$ software. Results were tested for differences in the bee mortality rate using the $\mathrm{chi}^{2}$-test on values at all-time intervals. The dose response curves obtained with SigmaPlot ${ }^{\circledR} 3.0$ software were the basis for the probit analysis and derivation of lethal dosage (LD) values. For the comparison of $\mathrm{pH}$ values of normally distributed data the $t$-test was used, alternatively in case of non-normally distributed data the Mann-Whitney rank sum test was used. The PER rates were compared between the groups for water responsiveness and within the groups for the sucrose solutions using chi $^{2}$-test and McNemar's test, respectively. The longevity data were analyzed using a Kaplan-Meier survival analysis, Gehan-Breslow. The density values of the bees in the small colony units were analyzed with the t-test. Regarding all statistical tests a difference was considered to be significant when the $p$-value obtained was lower than 0.05 . 


\section{Results}

\subsection{Laboratory Tests: Treatment of Individual Bees with OAD}

\subsubsection{Investigation of Lethal Effects-Acute Oral and Dermal Toxicity}

After dermal application of OAD the toxicity increased slowly during the observation time of 24 to $72 \mathrm{~h}$. After $72 \mathrm{~h}$ the application of 175 and $212.5 \mu \mathrm{g} /$ bee, respectively, showed no significant effect. After application of $250 \mu \mathrm{g}$ OAD the bee mortality was significantly higher (chi ${ }^{2}$-test, $p=0.003$ ) than at 175 and $212.5 \mu \mathrm{g} / \mathrm{bee}$. In the dosages 375 and $500 \mu \mathrm{g} /$ bee the mortality increased to $>20 \%$ (Figure 2). The NOAEL (72 h) for dermal application was 212.5, the LOAEL $250 \mu \mathrm{g}$ OAD/bee. The extrapolated $\mathrm{LD}_{10}(72 \mathrm{~h})$ is $256.4 \mu \mathrm{g} \mathrm{OAD} /$ bee. The $\mathrm{LD}_{10} 48 \mathrm{~h}$ after application reached a higher value than after $72 \mathrm{~h}: 467.7 \mu \mathrm{g} / \mathrm{bee}$. The $\mathrm{LD}_{10} 24 \mathrm{~h}$ after application exceeded this value but could not be determined from the data (Table 2).

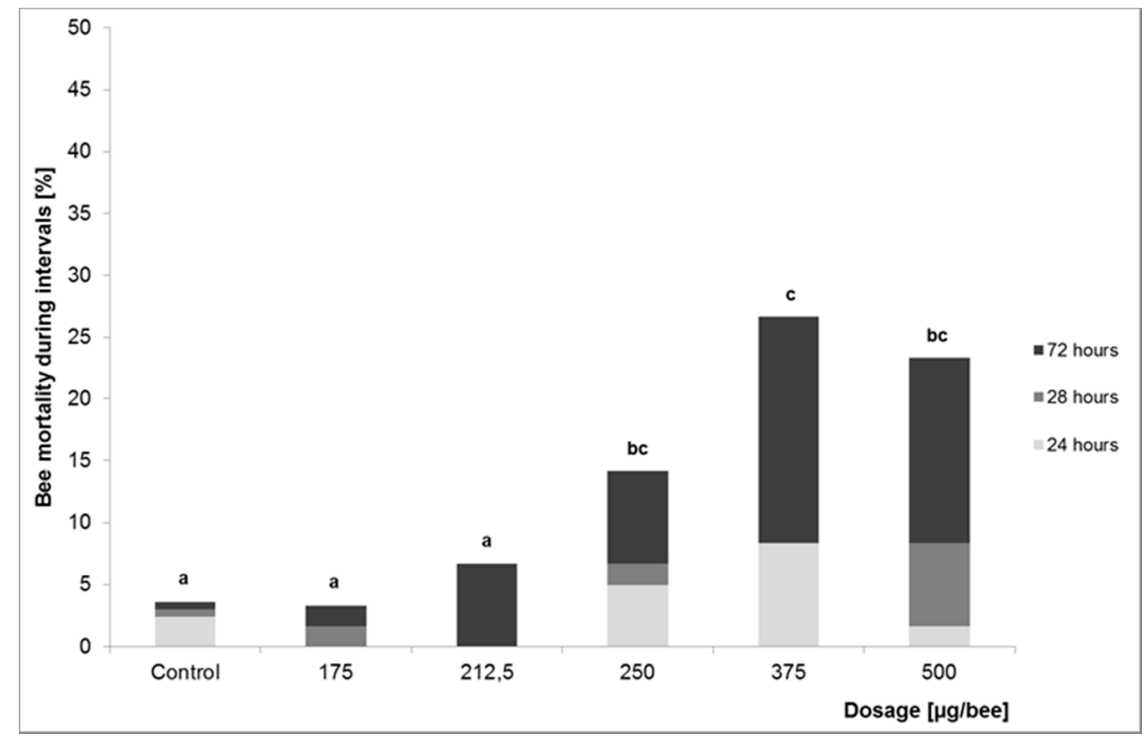

Figure 2. Bee mortality rates after dermal application of OAD during the three test intervals. Mortality rates with different lower-case letters are significantly different $\left(\right.$ chi $^{2}$-test, $\left.p \leq 0.05\right)$.

Table 2. Toxicity parameters after dermal and oral application of OAD.

\begin{tabular}{|c|c|c|c|c|}
\hline & \multicolumn{2}{|c|}{ Dermal Application ( $\mu \mathrm{g} / \mathrm{bee})$} & \multicolumn{2}{|c|}{ Oral Application ( $\mu \mathrm{g} / \mathrm{bee})$} \\
\hline & $48 \mathrm{~h}$ & $72 \mathrm{~h}$ & $48 \mathrm{~h}$ & $72 \mathrm{~h}$ \\
\hline $\mathrm{LD}_{10}$ & 467.7 & 256.4 & 68.1 & 60.3 \\
\hline NOAEL & n.d. $b$ & 212.5 & 75 & 50 \\
\hline LOAEL $^{a}$ & & 250 & 80 & 75 \\
\hline chi $^{2}$-test & n.d. ${ }^{b}$ & $p=0.003$ & $p<0.001$ & $p=0.027$ \\
\hline
\end{tabular}

${ }^{a}$ These values represent the lowest dosage with significant difference in bee morality compared to controls; ${ }^{\mathrm{b}}$ n.d. not defined, no significant differences occurred after $48 \mathrm{~h}$; $p$-values refers to the first significant increase in mortality (LOAEL).

After oral application, bee mortality occurred at relatively low concentrations compared to the dermal treatment (Figure 3). 10 and $50 \mu \mathrm{g}$ did not cause a mortality rate significantly different from the control group, while $75 \mu \mathrm{g}$ resulted in significantly higher bee mortality (chi ${ }^{2}$-test, $p=0.027$ ). A total of $100 \mu \mathrm{g}$ killed $55 \%$ of treated animals after $72 \mathrm{~h}$. The NOAEL (72 h) for oral application was 50, the LOAEL was $75 \mu \mathrm{g}$ OAD/bee. The $\mathrm{LD}_{10}$ obtained by probit analysis was $60.3 \mu \mathrm{g} / \mathrm{bee}$. The $\mathrm{LD}_{10} 48 \mathrm{~h}$ after application reached higher values than after $72 \mathrm{~h}: 68.1 \mu \mathrm{g} /$ bee. The $\mathrm{LD}_{10} 24 \mathrm{~h}$ after application was expected to exceed this value but this could not be determined from the data (Table 2). 


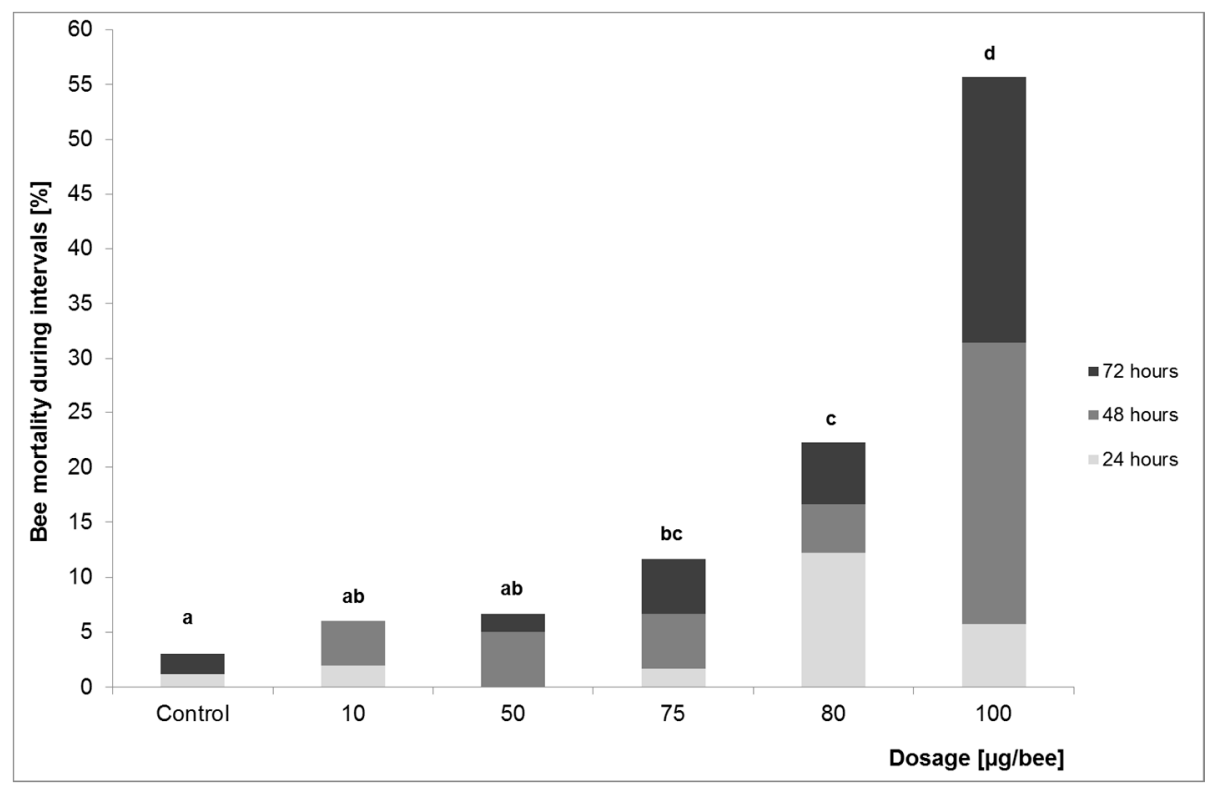

Figure 3. Bee mortality rates after oral application of OAD during the three test intervals. Mortality rates with different lower-case letters are significantly different $\left(\right.$ chi $^{2}$-test, $\left.p \leq 0.05\right)$.

During the experiment, changes in behavior were observed only after oral application of dosages also causing increased mortality within this period ( $\geq 75 \mu \mathrm{g} / \mathrm{bee}$ ). The bees were less active, showed minor movement and slowly formed bee clusters at the top of the cage. Shortly after the application they showed increased self-grooming. Some bees also extended the proboscis.

\subsubsection{Investigation of Sublethal Effects}

\section{Responsiveness to Water and Ascending Concentrations of Sucrose Solution}

The PER on water increased after the treatment in the test (McNemar's test, $p \leq 0.001$ ) and control bees (McNemar's test, $p \leq 0.01$, Figure 4). However, bees treated with OAD showed significant higher response rates to water than the controls $\left(\mathrm{chi}^{2}\right.$-test, $\left.p \leq 0.001\right)$.

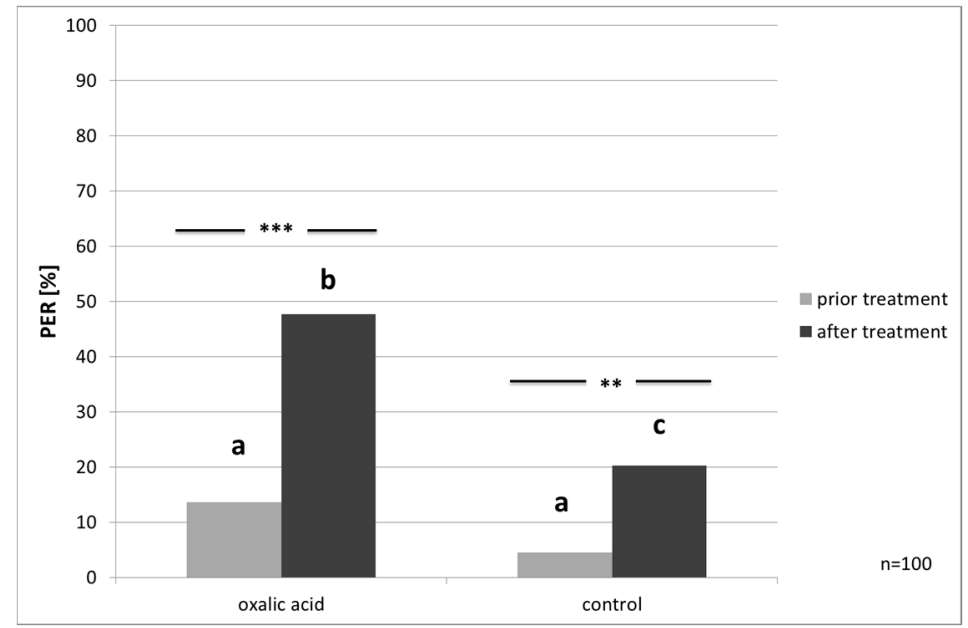

Figure 4. PER (Proboscis Extension Response) to water prior and after treatment: both groups show increased responsiveness after the treatment (McNemar's test, $p \leq 0.001$ and $p=0.004$,). Different lower-case letters indicate significant differences between groups, before and after treatment (chi ${ }^{2}$-test, $p \leq 0.001$ ) additionally; significant differences are marked with asterisks'. 
The sucrose responsiveness was also influenced by treatments, in the control group the responsiveness decreased significantly at $3 \%$ and $10 \%$ concentration (McNemar's test, $p \leq 0.025$, Figure 5).

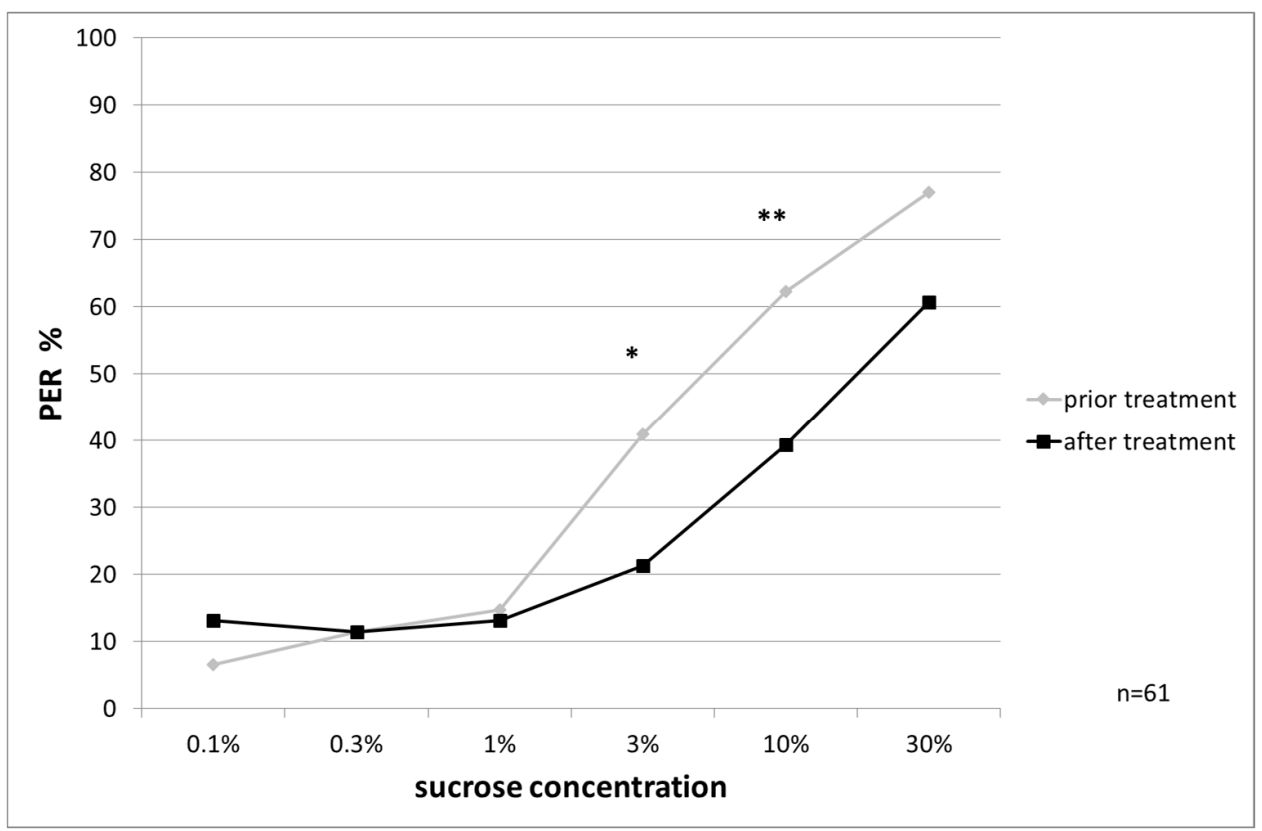

Figure 5. PER to ascending sucrose concentrations from control bees: response is significantly decreased at $3 \%$ and $10 \%$ concentration (McNemar's test, $p=0.025$ and $p=0.018$ ), significant differences are marked with asterisks'.

Bees treated with OAD showed increased sucrose responsiveness, significantly at $0.1 \%$ (McNemar's test, $p=0.024$, Figure 6).

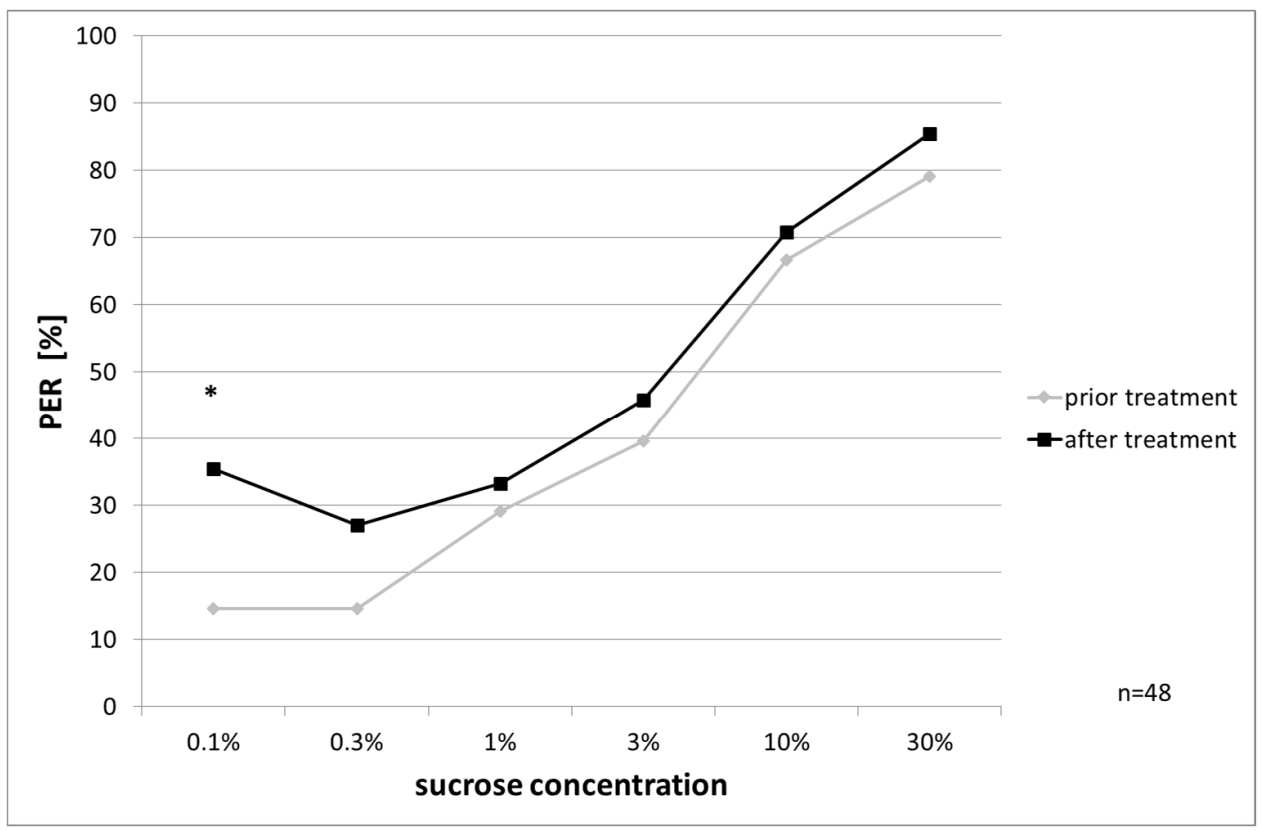

Figure 6. PER to ascending sucrose concentrations from OAD-treated bees: response after treatment is significantly increased at $0.1 \%$ concentration (McNemar's test, $p=0.024$ ), significant differences are marked with asterisks'. 
Longevity under Laboratory Conditions

The highest proportional bee mortality occurred directly after the treatment (test group) and between the 20th and 21st day after hatching (control group). In the test group bees lived at least for two days, for a maximum of 31 days and on average for 6.4 days. The bees in the control group lived for an average of 24.4 days (min. two days, max. 33 days). This can be demonstrated in the trend of the survival curves (Kaplan-Meier survival analysis, Gehan-Breslow, $p \leq 0.001$, Figure 7).

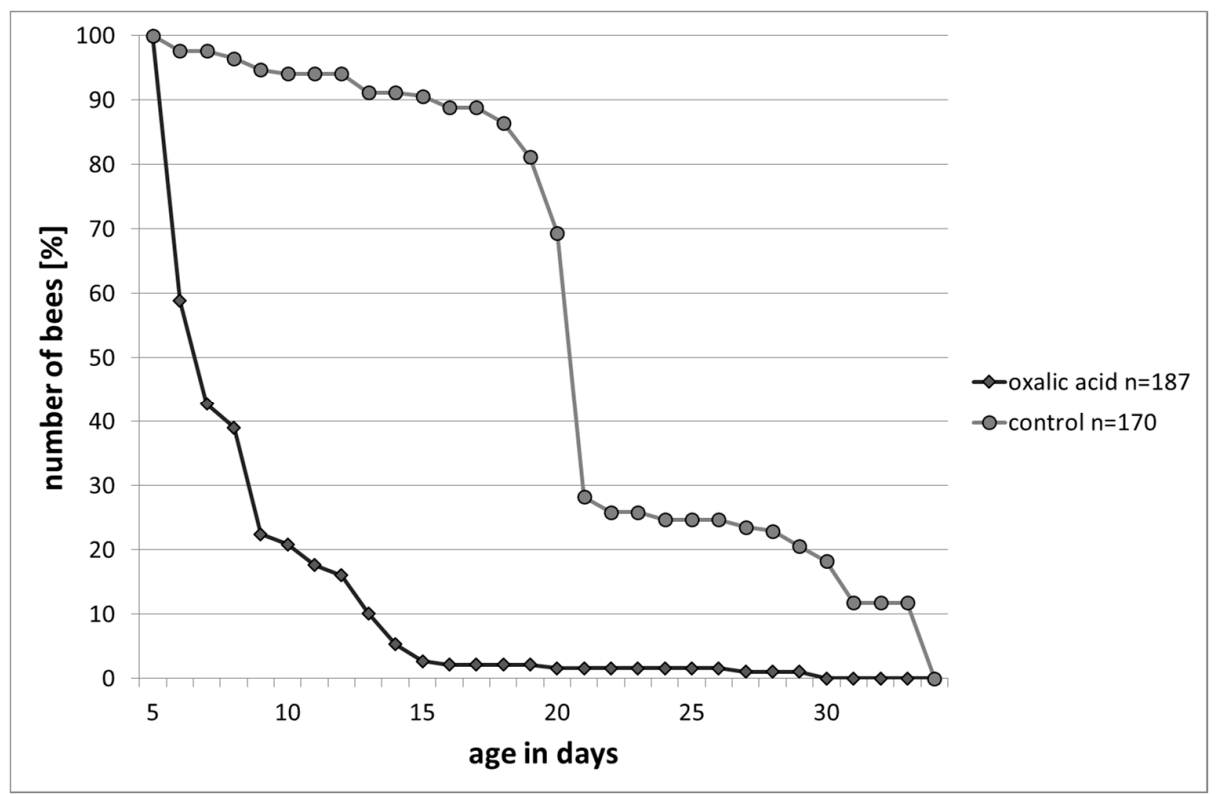

Figure 7. Survival curves under laboratory conditions: bees in the control group survived significantly longer than bees treated with OAD (Kaplan-Meier survival analysis, Gehan-Breslow, $p \leq 0.001$ ).

$\mathrm{pH}$ Values of the Digestive System and the Hemolymph

Crop: The $\mathrm{pH}$ values of the crops' content after oral OAD application were subject to slight variation (Figure 8$)$. The average $\mathrm{pH}$ value $( \pm \mathrm{SD})$ after oral application of OAD $(24 \mathrm{~h}: 4.62 \pm 0.4 ; 48 \mathrm{~h}$ : $4.64 \pm 0.36 ; 72 \mathrm{~h}: 4.44 \pm 0.43$ ) was lower during $72 \mathrm{~h}$ than the control. After the dermal application, the $\mathrm{pH}$ values were at $24 \mathrm{~h}: 4.82 \pm 0.37$ and $48 \mathrm{~h}: 5.02 \pm 0.4$ remaining at the control level. Only during the last interval did the $\mathrm{pH}$ subside to $4.49 \pm 0.38(72 \mathrm{~h})$. The $\mathrm{pH}$ values obtained by the control group were $24 \mathrm{~h}: 4.86 \pm 0.46 ; 48 \mathrm{~h}: 4.80 \pm 0.44$; and $72 \mathrm{~h}: 4.99 \pm 0.92$. However the differences were not significant (Mann-Whitney rank sum test, $p \geq 0.05$ ), and the standard deviation of means is comparatively high.

Ventriculus: The average $\mathrm{pH}$ values of the liquid ventriculus contents were significantly reduced between 24 and $48 \mathrm{~h}$ after oral application of OAD: $6.50 \pm 0.24(24 \mathrm{~h})$ and $6.38 \pm 0.32(48 \mathrm{~h}$, Mann-Whitney-rank-sum-test, $p=0.002$ ), respectively (Figure 9). During the last interval, the $\mathrm{pH}$ value averaged $6.59 \pm 0.17(72 \mathrm{~h})$. After the dermal application of $\mathrm{OAD}$, the $\mathrm{pH}$ value was on average $6.52 \pm 0.17(24 \mathrm{~h})$. Within the following intervals the $\mathrm{pH}$ reduced significantly to $6.40 \pm 0.21(48 \mathrm{~h})$ compared with the control group (t-test, $p=0.002$ ) and $6.48 \pm 0.14$ after $72 \mathrm{~h}$ (t-test, $p=0.028$ ). The $\mathrm{pH}$ values obtained by the corresponding control groups remained at a constant level: $24 \mathrm{~h}: 6.55 \pm 0.2$; 48 h: $6.55 \pm 0.21 ; 72$ h: $6.55 \pm 0.16$.

Rectum: The $\mathrm{pH}$ value of the liquid rectum contents averaged $4.98 \pm 0.2724 \mathrm{~h}$ after oral OAD application (Figure 10). After $48 \mathrm{~h}$, the $\mathrm{pH}$ was significantly reduced to $4.77 \pm 018$ (t-test, $p<0.001$ ); after $72 \mathrm{~h}$, the $\mathrm{pH}$ reached $4.83 \pm 0.16$ and remained significantly different to the control group (t-test, $p=0.003)$. After dermal application of $\mathrm{OAD}$, the $\mathrm{pH}$ value averaged $5.09 \pm 0.16(24 \mathrm{~h})$ and $5.11 \pm 0.18$ $(48 \mathrm{~h})$. During the last interval, the $\mathrm{pH}$ was $5.10 \pm 0.17$ and significantly different to the control group 
value (t-test, $p=0.016)$. The $\mathrm{pH}$ values obtained by the corresponding control groups were: $24 \mathrm{~h}$ : $5.05 \pm 0.31 ; 48$ h: $5.05 \pm 0.24 ; 72$ h: $4.99 \pm 0.24$.

Hemolymph: OAD, $24 \mathrm{~h}$ after oral application, caused a significant reduction in the average $\mathrm{pH}$ value of the hemolymph with $6.72 \pm 0.31$ compared to the control group ( $6.98 \pm 0.17$; Mann-Whitney rank sum test, $p=0.011$, Figure 11). During the remaining intervals, the $\mathrm{pH}$ increased to $6.83 \pm 0.22$ $(48 \mathrm{~h})$ and $6.97 \pm 0.27(72 \mathrm{~h})$. After dermal application, the $\mathrm{pH}$ values measured at the first and second intervals were $7.00 \pm 0.12(24 \mathrm{~h})$ and $7.00 \pm 0.08(48 \mathrm{~h})$, respectively, and thus similar to the control groups: $6.98 \pm 0.17(24 \mathrm{~h}) ; 6.96 \pm 0.26(48 \mathrm{~h})$. After $72 \mathrm{~h}$, the average $\mathrm{pH}$ value was $6.88 \pm 0.15$, significantly reduced in comparison to the control group $7.17 \pm 0.21$ (t-test, $p=0.006$ ).

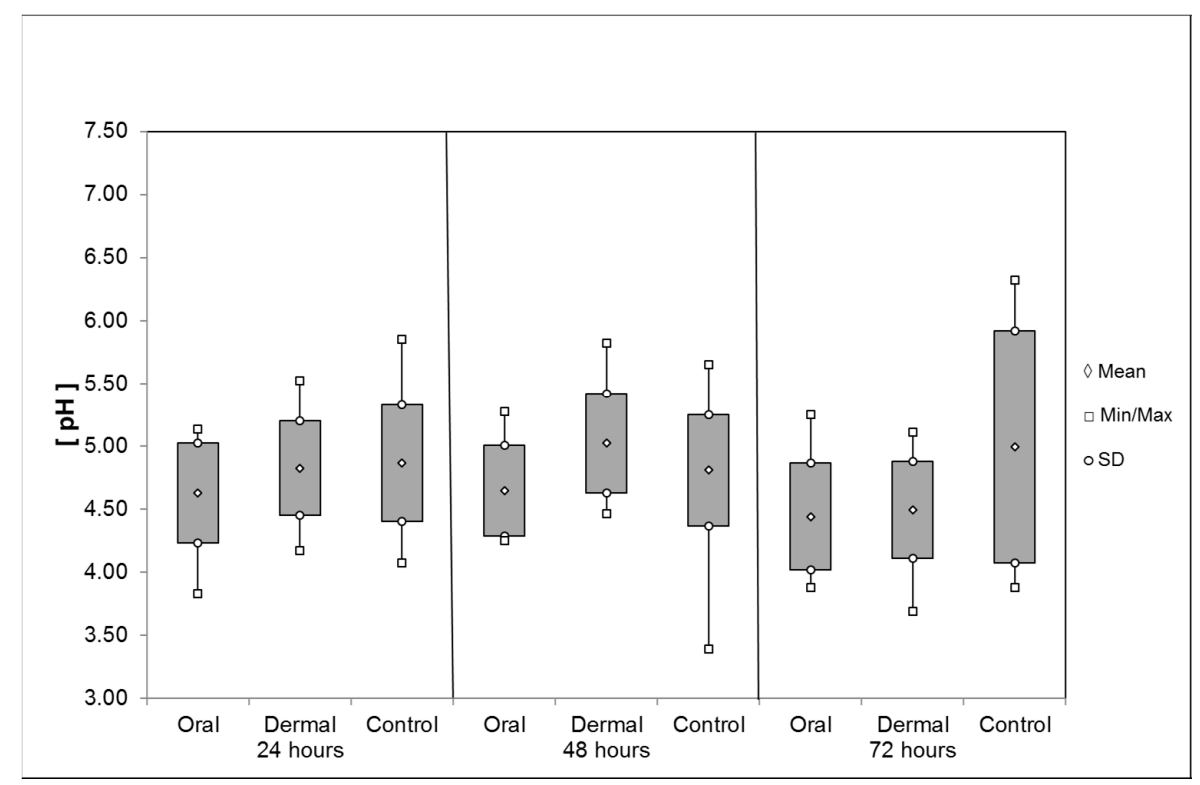

Figure 8. Average $\mathrm{pH}$ of the liquid contents of the honeybee crop after oral and dermal treatment (n $\leq 52 /$ group).

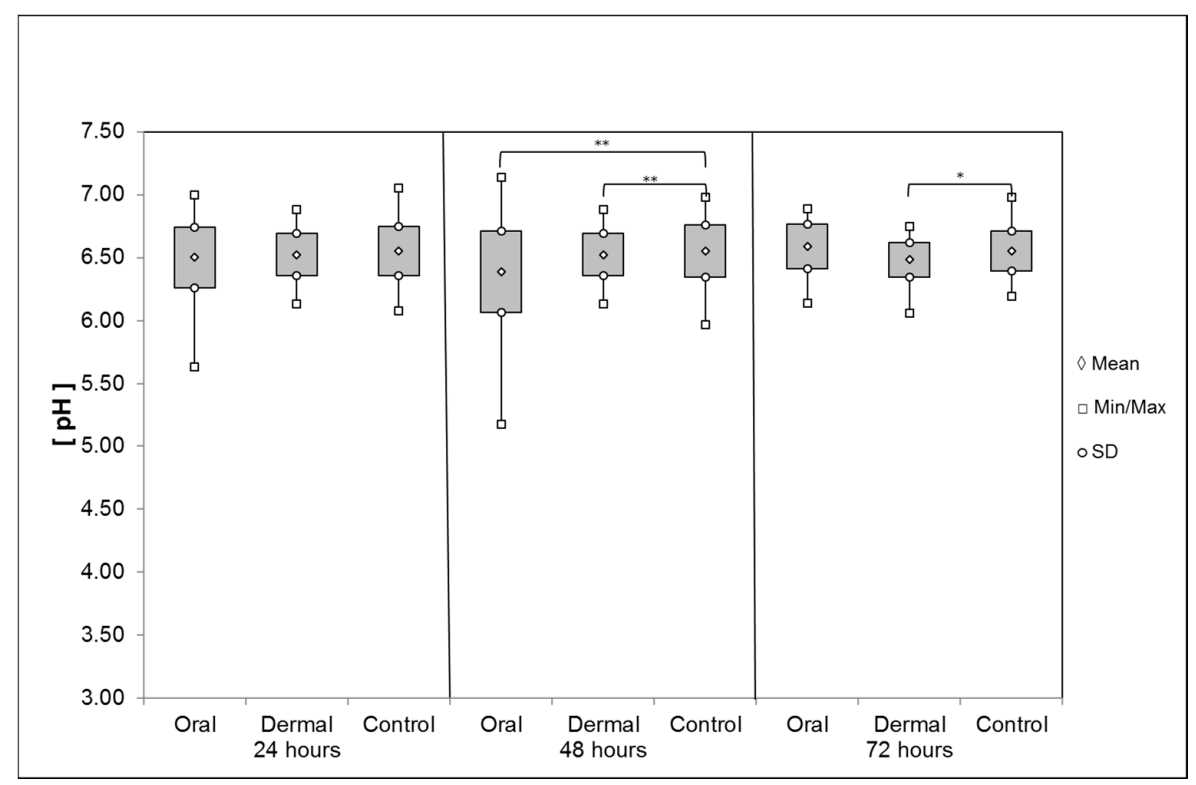

Figure 9. Average $\mathrm{pH}$ of the ventriculus contents after oral and dermal treatment ( $\mathrm{n} \leq 68$ /group). Significant differences are indicated by asterisks (48 h: Mann-Whitney rank sum test, $p=0.002 ; 72 \mathrm{~h}$ : t-test, $p<0.05)$; significant differences are marked with asterisks'. 


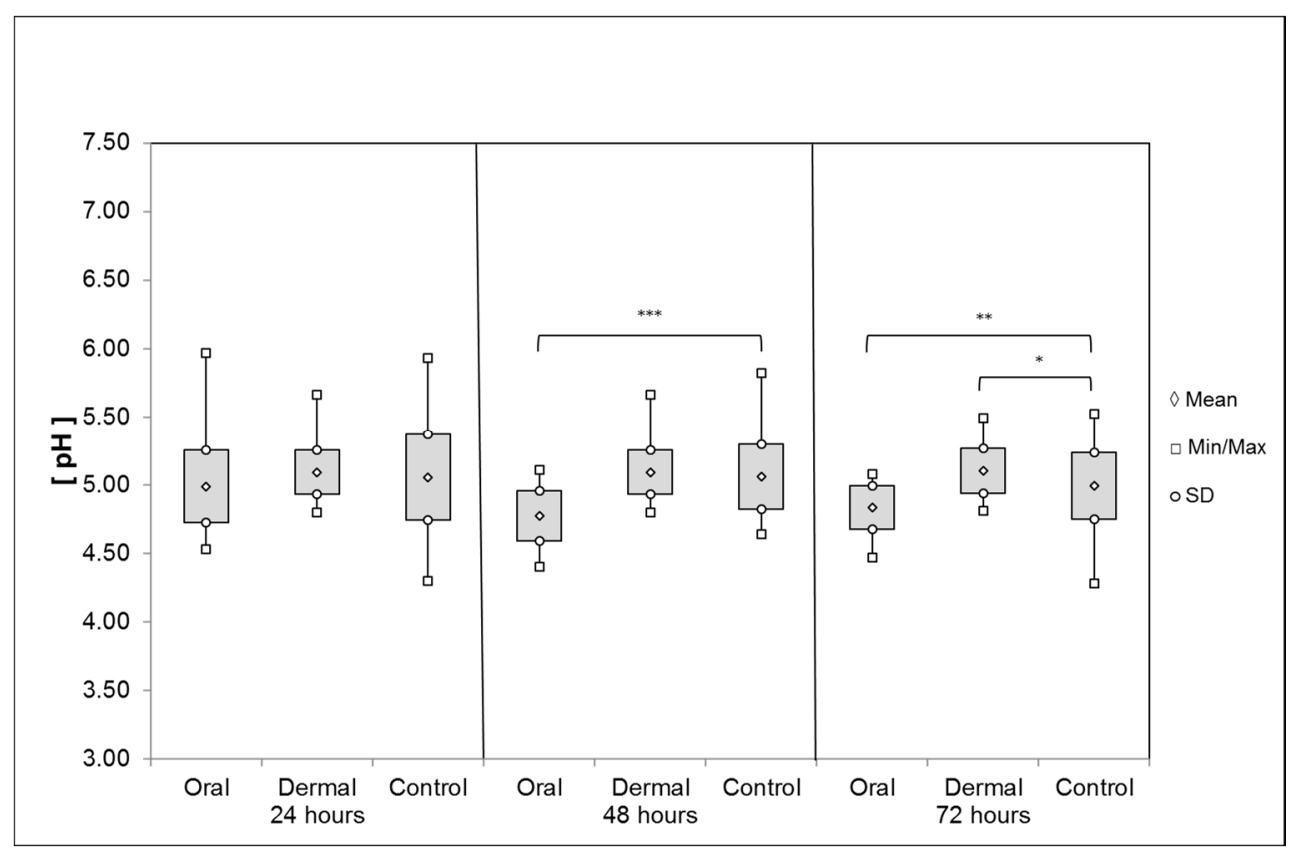

Figure 10. Average $\mathrm{pH}$ of the rectum contents after oral and dermal treatment ( $\mathrm{n} \leq 68$ /group). Significant differences are indicated by asterisks ( $48 \mathrm{~h}$ : t-test, $p=0.001 ; 72 \mathrm{~h}$ : t-test, $p<0.05)$; significant differences are marked with asterisks'.

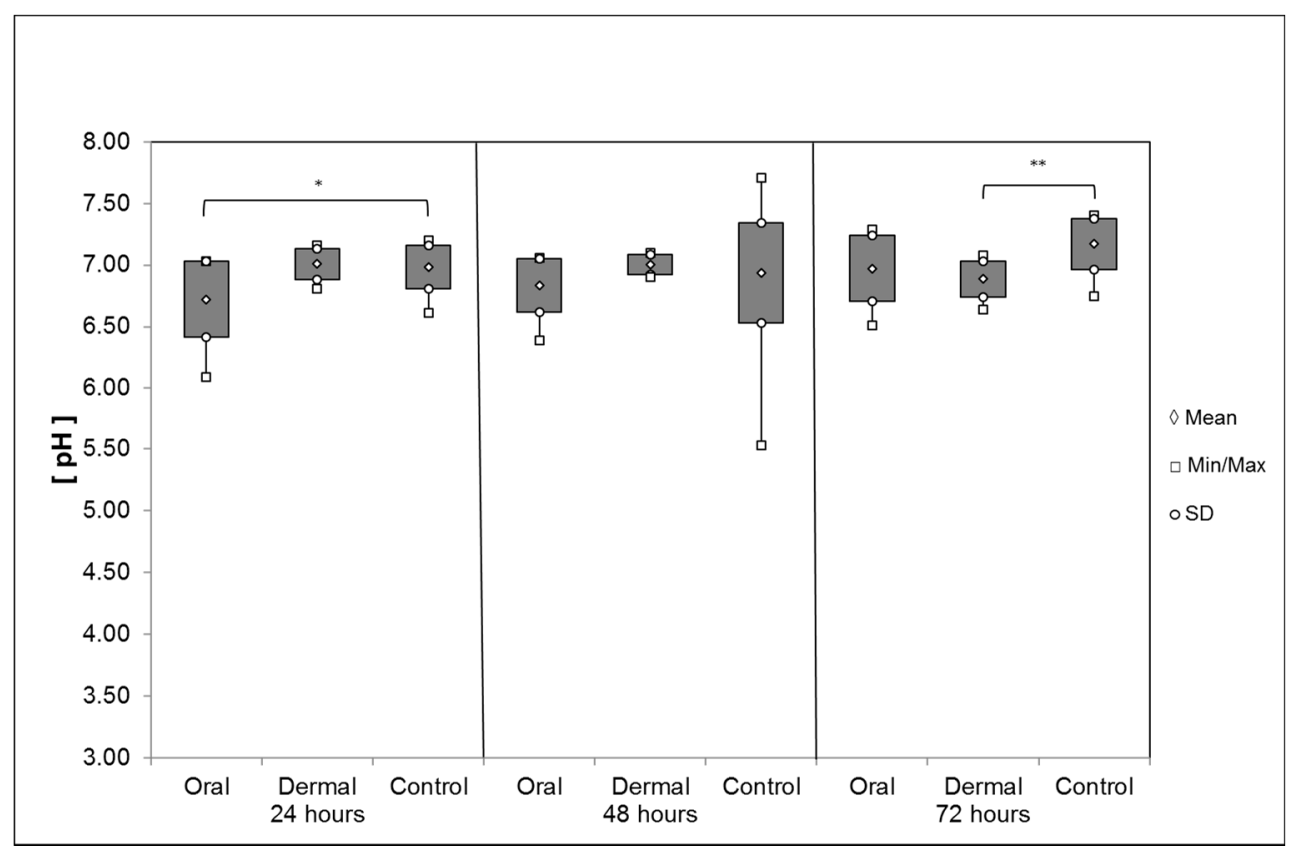

Figure 11. Average $\mathrm{pH}$ of the hemolymph after oral and dermal treatment ( $\mathrm{n} \leq 26$ /group). Significant differences are indicated by asterisks' (24 h: Mann-Whitney rank sum test, $p=0.011$; $72 \mathrm{~h}$ : t-test, $p=0.006)$; significant differences are marked with asterisks'.

\subsection{Computer Tomography of Honey Bee Colonies: Distribution of OAD}

The distribution of OAD in the colony after topical application was demonstrated by macroCT scanning (Figure 12). The control measurements achieved a mean density value of $-219.77 \pm 93.3 \mathrm{HU}$. The relatively high standard deviation was caused by the heterogeneity of the bees' body mass. After application of OAD $(10 \mathrm{~min})$ the density value increased to a mean value of $-98.97 \pm 87.06 \mathrm{HU}$, which 
was significantly different from the controls (t-test, $p \leq 0.001$ ). Thirty minutes after application the mean value was $-134.98 \pm 89.5 \mathrm{HU}$, significantly different compared to the controls and the values achieved after $10 \mathrm{~min}$ (t-test, $p \leq 0.001$, Figure 13).

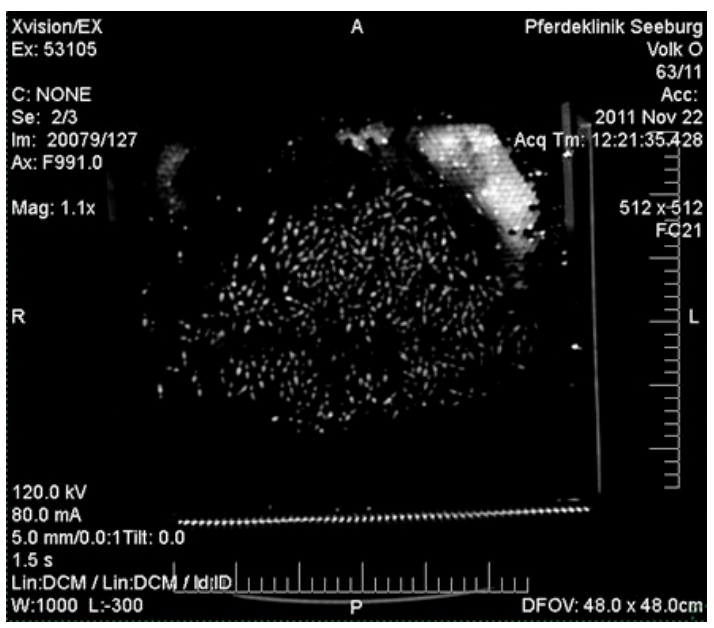

Figure 12. MacroCT scanner image: bees on the comb, $30 \mathrm{~min}$ after application of OAD and Unilux. Visualization of a comb with bees sitting on it: dark areas show low densities and bright areas show high densities.

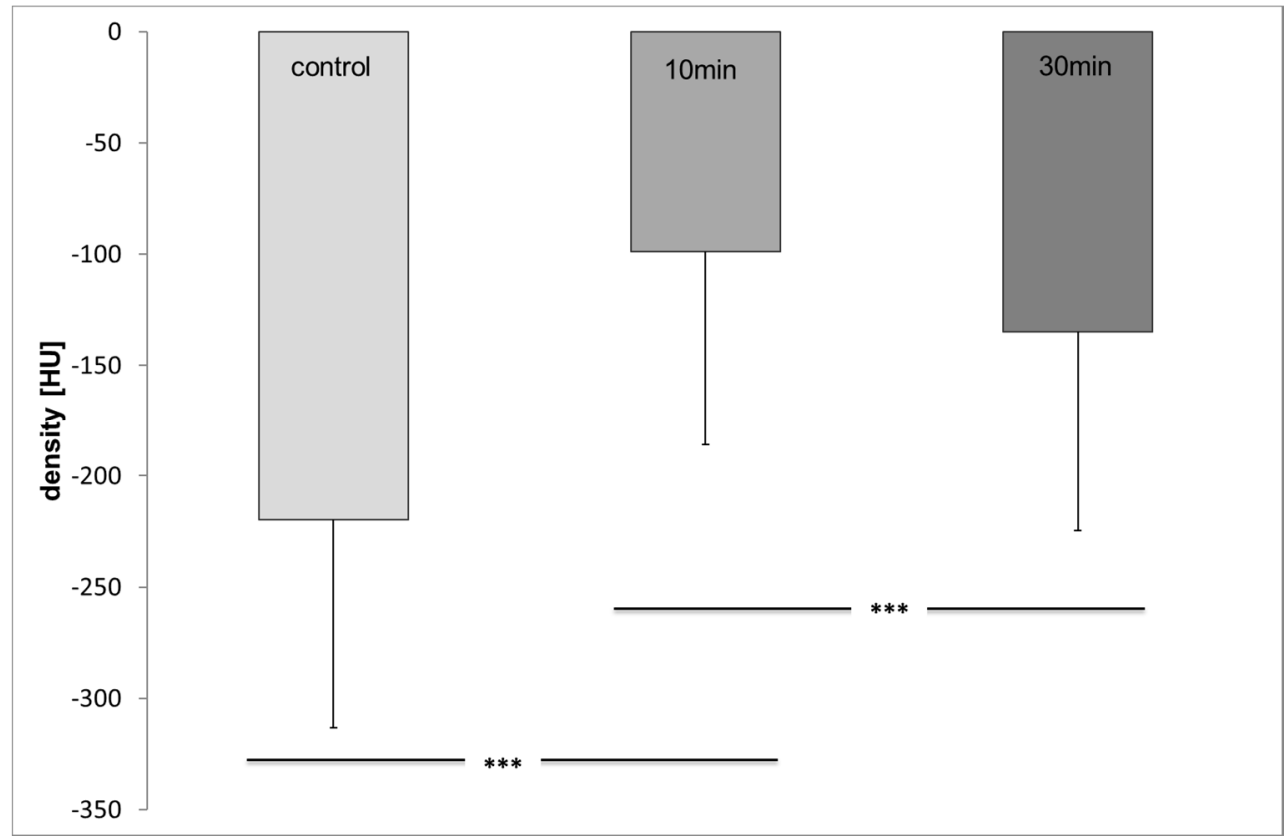

Figure 13. Density values before (control) and 10 and $30 \mathrm{~min}$ after treatment ( $\leq 144$ /group; t-test, $p \leq 0.001)$, significant differences are marked with asterisks'.

The mean value $(-96.03 \pm 87 \mathrm{HU})$ in the central area of the combs $10 \mathrm{~min}$ after treatment of the bees was comparable to the bees in the boundary area $(-101.92 \pm 86.7 \mathrm{HU})$. However, the mean density values thirty min after the treatment were significantly different compared to the central area $(-153.04 \pm 77.8)$ and boundary area $(-116.9 \pm 97.1)$ of the combs (t-test, $p=0.015$, Figure 14). 


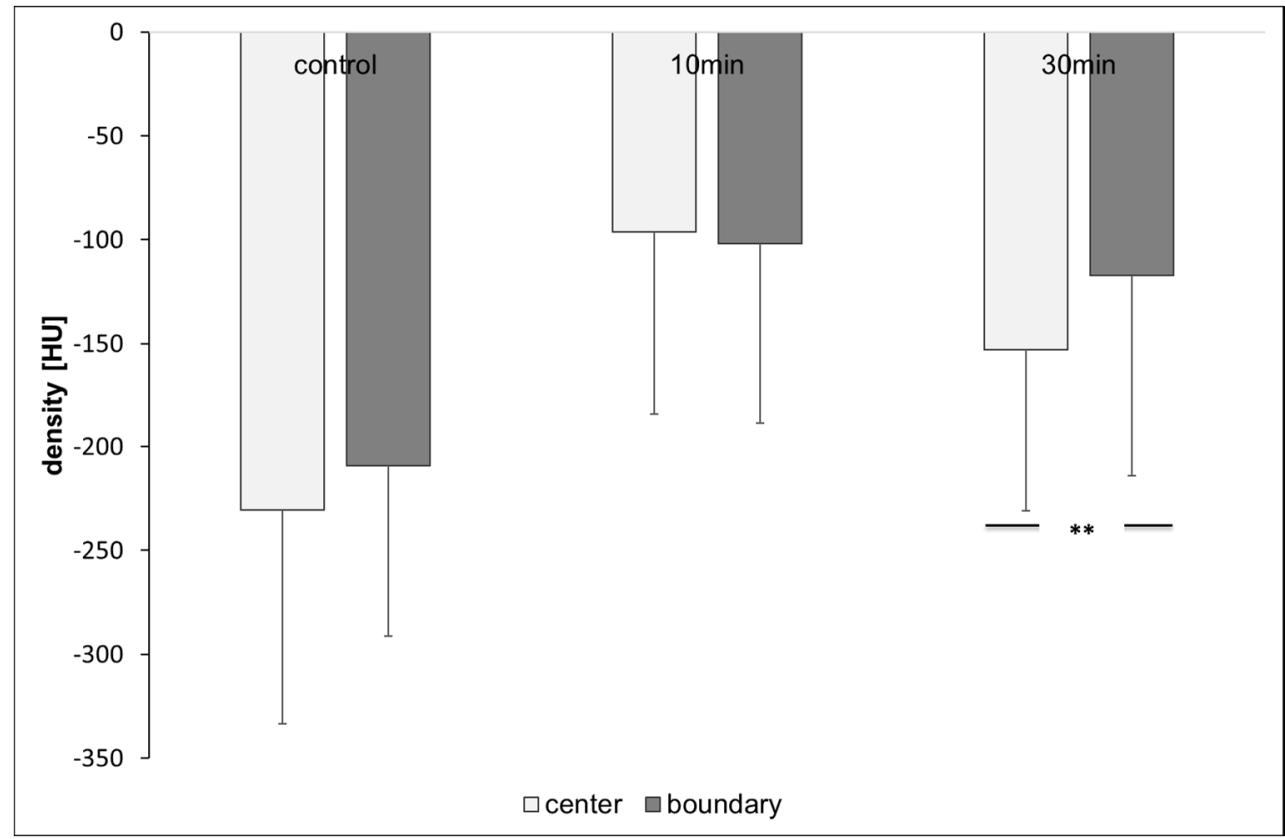

Figure 14. Density values of the comb areas center and boundary: significant differences between areas occur 30 min after treatment ( $\leq 72$ /group; t-test, $p=0.015)$, significant differences are marked with asterisks'.

In the colony examined for the long-term distribution, the control measurements achieved a mean density value of $-200.61 \pm 86.87 \mathrm{HU}$. Three days after the application of OAD the density value increased to a mean value of $-128.1 \pm 89.92 \mathrm{HU}$ and was significantly different from the control measurement (t-test, $p \leq 0.001$, Figure 15). Seven and 14 days after application the mean value was $-151.61 \pm 77.14 \mathrm{HU}$ and $-158.46 \pm 78.32 \mathrm{HU}$, respectively, significantly different compared to the controls and the values achieved after three days (t-test, $p \leq 0.001$, Figure 15).

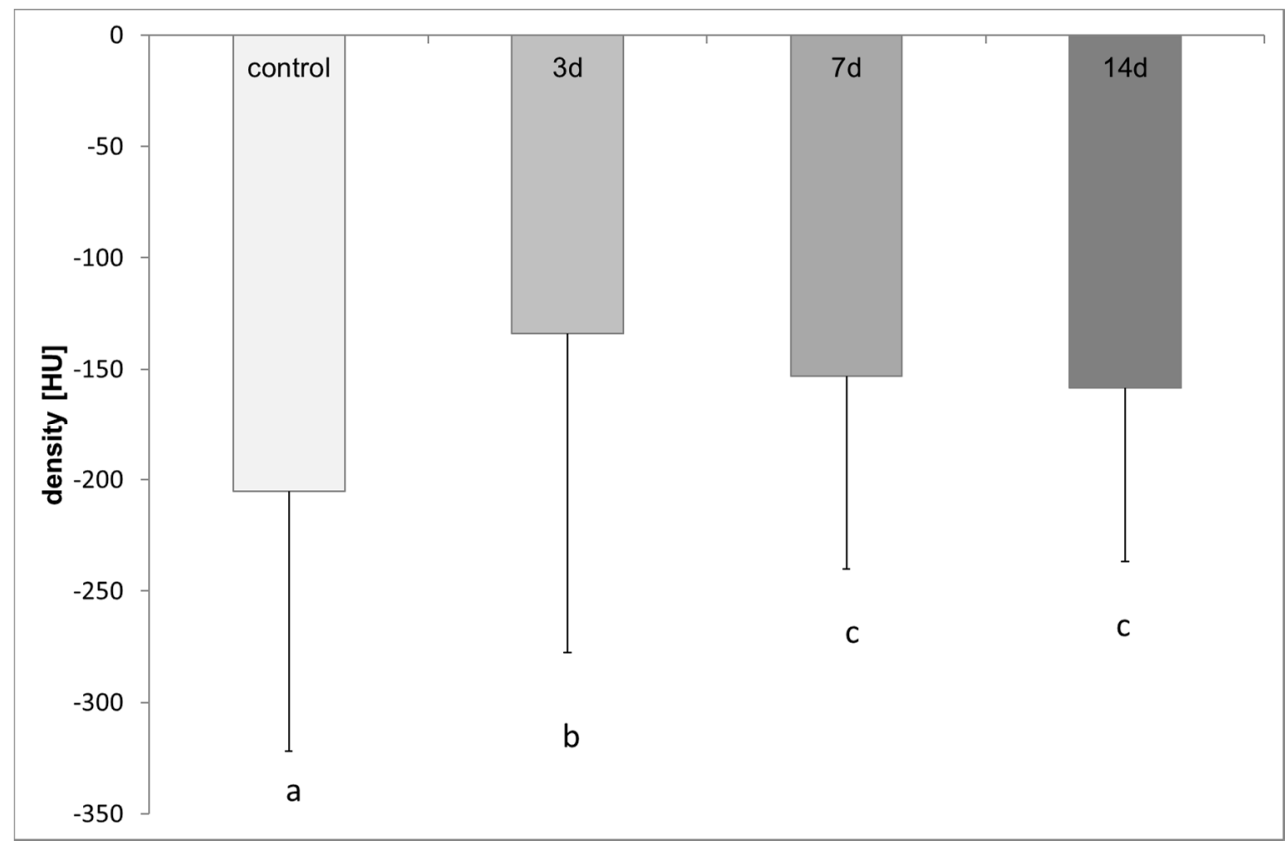

Figure 15. Density values before (control) and 3, 7 and 14 days after treatment: different lower-case letters indicate significant differences ( $\mathrm{n} \leq 211$ /group; t-test, $p \leq 0.001$ ). 


\section{Discussion}

We found unequal results of OAD toxicity after dermal and oral application-ingestion of OAD is much more toxic to bees than application on the cuticula, which was relatively well tolerated. This corresponds with our first report of bee tolerability concerning OAD as a single compound [10].

Investigations of toxicity are often conducted with combined substances. This makes it difficult to compare these results with our findings. Concerning the dermal application of the toxic effects of different dosages are reported in the literature. Dosages $<100 \mu \mathrm{g} /$ bee did not cause significant mortality after $48 \mathrm{~h}$, this is less than half the dosage of $212.5 \mu \mathrm{g} /$ bee with no observed mortality in our test. The described $\mathrm{LD}_{10}(48 \mathrm{~h})$ of $176.68 \mu \mathrm{g} /$ bee was derived from a combinatory effect of OAD and acetone on anaesthetized worker bees $\left(\mathrm{CO}_{2}\right)$ [11]. It was lower than the $\mathrm{LD}_{10}(48 \mathrm{~h})$ of OAD as a single agent in our trial, which reached a level of $467.7 \mu \mathrm{g} /$ bee. A combinatory effect of OAD and acetone on anaesthetized worker bees $\left(\mathrm{CO}_{2}\right)$ may explain the explicitly higher responsiveness of the tested animals in the cited trials compared to our findings. Other tests with a polyhybrid subspecies of A. mellifera resulted in a level without observed effect of $400 \mu \mathrm{g} /$ bee and a lowest observed effect level of $600 \mu \mathrm{g} /$ bee after $48 \mathrm{~h}$ [12].

In our experiments $175 \mu \mathrm{g} /$ bee, corresponding to the $3.5 \%$ solution (30-50 mL depending on colony size) used in beekeeping practice, did not cause mortality for individual bees, different from controls $72 \mathrm{~h}$ after dermal application. Oral application resulted in high bee mortality at relatively low concentrations compared to the dermal treatment. Bees reacted much more sensitively to the oral application of OAD. The LOAEL for dermal application is higher than for oral application by a factor $>3$.

To assess risk of an applied substance, not only mortality but also the effects on physiological processes and behavior after pesticide exposition must be considered as described [13]. Physiological effects concerning acetylcholinesterase and glutathione S-transferase activities, as described for exposure to fluvalinate, are not documented after OAD treatment ( $3 \%$ in $32 \%$ sucrose solution $w / w$, $50 \mathrm{~mL} /$ colony) on pupae, newly emerged, nursery and forager bees [14,15]. Honey bee larvae, treated with OAD by spraying (about $121 \mu \mathrm{g}$ solution/larvae) undergo histologic changes-accidental cell death leading to necrosis [16]. These effects may be causally responsible for the brood death after application of OAD (3\% in 50\% sucrose solution, two treatments during summer) in breeding colonies [17]. In beekeeping practice, acute damages to the brood can be excluded when honeybee colonies are treated with OAD during the brood free period. However, long term effects are possible; OAD has been found in bee colonies even six months after topical application following regular treatment [18]. Long-term effects (up to four months after application) like a reduced amount of brood in treated colonies (3\% OAD $w / v, 4 \mathrm{~mL}$ per comb side, spraying) have been reported [19].

After dermal and oral application, respectively, of high dosages up to $1320 \mu \mathrm{g} / \mathrm{bee}$, OAD was recovered in the internal organs and the hemolymph of adult bees [20,21]. It is assumed that after dermal application the cuticula can be penetrated by the acid [21]. Also, pathological repercussions e.g., degeneration of rectal epithelium, malphigian tubules and ventriculus, have been described after dermal application [20]. $C^{14}$-marked OAD could be found in the abdominal structures of worker bees after topical application into the colony [22].

The treatment with OAD caused sublethal effects on A. mellifera. First indications of changes in the $\mathrm{pH}$ of internal organs and the hemolymph after a single dermal application of OAD with a dosage of $175 \mu \mathrm{g} /$ bee have been provided [23]. The individual treatment of honeybees with OAD in our experiments changed the $\mathrm{pH}$-value of the intestinal parts and the hemolymph. The $\mathrm{pH}$ values of the crop contents reflect the acid application, specifically after oral ingestion. Significant differences could be found in the $\mathrm{pH}$ values of the ventriculus $48 \mathrm{~h}$ after oral and $72 \mathrm{~h}$ after dermal treatment: These deferred reductions in the tested time intervals could be caused by the slower penetration of the acid through the cuticula compared to the direct oral intake. Differences in the $\mathrm{pH}$ structure of the rectum and the hemolymph were also found, the $\mathrm{pH}$ reduction occurs faster after oral application. The proof of a $\mathrm{pH}$ reduction in the digestive system even after $72 \mathrm{~h}$ indicates a long disposition of OAD 
in the bee. The returned balance of the $\mathrm{pH}$ measured in the hemolymph $48 \mathrm{~h}$ after oral application also indicates a possible buffer capacity of the hemolymph.

We could demonstrate that $\mathrm{OAD}$ moving through the digestive system or penetrating the cuticula modified the $\mathrm{pH}$ structure of the honeybee's intestinal parts and the hemolymph. The shift towards stronger acidity after OAD treatment supports that damage to the epithelial tissue and organs [20] may be caused by hyperacidity. It also corresponds to the timescale of recovered OAD after oral application [21]. The increased acidity can cause chemical burns which eventually lead to necrosis [16], however a threshold cannot be derived from our tests due to the low dose applied.

Further sublethal effects could be demonstrated in a decreased longevity under laboratory conditions and increased responsiveness to water. Bees treated with OAD died much sooner than bees treated with sucrose solution. The increased responsiveness to water $24 \mathrm{~h}$ after OAD treatment indicated an acidosis of the bees, which they may compensate with an increased uptake of water. This assumption is supported by the shift towards stronger acidity, found-after OAD treatment conducted-in the measurements of the $\mathrm{pH}$-values. These results indicate a general impairment of the bees after treatment. The treatment in autumn/winter affected primarily long-living winter bees which are essential for winter survival and successful colony development in the spring. Treatment during the summer with brood can lead to substantial brood damage [15] as described above. Even by treating artificial swarms or nucleus colonies, it cannot be certain that damages will not occur due to the long-term exposure to OAD in the colony $[18,19]$.

It has been described that after dermal administration bees carry white deposits primarily on the body [11,20] and later also on the third pair of legs [24]. Concerning sublethal effects in the colony after individual dermal application of $175 \mu \mathrm{g} / \mathrm{bee}$, changes in behavior were found [24]: the bees were less active, showed reduced brood care, increased grooming behavior, and they also had a reduced life span.

In beekeeping practice OAD is mostly applied topically. The distribution in the colony may occur on two pathways: (1) through oral intake and distribution via trophallaxis; and/or (2) contact between bees and also contaminated hive material. OAD vapors in the colony seem to be of minor importance as OAD has a low volatility [25]. However, the colony treatment using 3.5\% OAD solution ( $30-50 \mathrm{~mL}$ per colony, depending on colony strength, applied by trickling) corresponds to an individual dosage of approximately $175 \mu \mathrm{g} \mathrm{OAD} / \mathrm{bee}$. This dosage is well tolerated by individual bees in the laboratory and is well below the lowest observed adverse effect level concerning mortality after dermal application in our test.

OAD applied orally in the laboratory causes high bee mortality. As the mortality observed in the treated colonies was not highly increased, OAD was probably ingested in relatively low amounts by grooming and/or trophallaxis. The oral intake of OAD after treatment at the colony level seems to be of little importance. The threshold value derived in the laboratory was therefore not exceeded for most of the target animals. This corresponds with the findings that when OAD is applied in a solution with high sugar content the bee mortality increases [26]. This suggests that high sugar content leads to more ingestion. In laboratory trials, significantly higher bee mortality was found when sugar was added to the OAD solution [27].

In practical beekeeping, appropriate use of OAD (on average $175 \mu \mathrm{g} /$ bee) by topical application in the field is relatively safe for A. mellifera on the colony level, even when some individuals die. Based on our findings in the laboratory, the threshold with first adverse effects (LOAEL) could be reached in the colony when an overdose of $43 \%$ for the individual bee is applied. However, due to the attractiveness of sucrose solutions to bees they ingest the solution even if it contains toxic substances. A sugar substitute e.g., glycerol, with a high viscosity and desirable distribution can prohibit oral uptake by the bees [9]. It could optimize the application of OAD, but so far it has not been approved as a veterinary drug ingredient for bees in combination with OAD.

High acaricidal efficacy after topical application was only found when bee to bee contact took place [11]. In further trials, when body contact between the bees was prevented, but trophallaxis 
allowed, it has been shown that trophallactic interaction did not lead to mite mortality; bee to bee contact seemed to be the primary route of distribution of OAD in acaricidal relevant dosages [28].

Due to only minor oral intake, systemic efficacy against $V$. destructor seems to be improbable. A systemic effect requires a transfer of the acid into the hemolymph of the bee. OAD was found in the hemolymph only after application of very high dosages $[20,21]$. After application of the 3.5\% OAD solution ( $175 \mu \mathrm{g} / \mathrm{bee})$, as used in beekeeping, changes in the $\mathrm{pH}$ structure were found, but OAD was not recovered in the hemolymph of single bees at a detection limit of $2.5 \mu \mathrm{g}$ [24]. This amount used in beekeeping practice seems to be too low to reach a systemic toxicity to $V$. destructor through ingestion. Therefore, we conclude that the mode of action in the colony must be contact poisoning against $V$. destructor.

In order to reach high efficacy, the ingredient acting by contact must be distributed in the colony. The distribution of OAD was shown by macroCT. The results of the roentgenoscopy showed high density values for the individual bees in the test, much higher than in the control measurement. A good distribution was already achieved after $10 \mathrm{~min}$; this could be documented in the central and boundary areas of the combs. Lower density values in the central comb areas compared to the boundary regions obtained after thirty minutes reflected the movement of the bees. After thirty minutes the density was generally lower, which led us to the assumption that OAD was now also spread to the material, e.g., the wall of the hive. Bees have constant contact with the hive material; therefore, OAD can be distributed again onto the bees, maintaining a long-term contact with the acid. OAD on hive material can be found even several months after application [18]. The macroCT analysis demonstrated a rapid and consistent distribution of OAD involving a reduction of the individual dosage over time. Even after 14 days, the density of the bees was still significantly higher than prior to treatment, indicating a potential efficacy of at least up to 14 days. The results from the field trials, where the maximum efficacy against mites was reached ten days after treatment, support this assumption [29].

\section{Conclusions}

OAD used to treat varroosis of $A$. mellifera shows a rapid and consistent distribution in the colony for at least up to 14 days, and high efficacy against the mite, but also lethal and sublethal effects. In practical beekeeping, appropriate use of OAD (one topical application, on average $175 \mu \mathrm{g} / \mathrm{bee}$ ) is relatively safe for $A$. mellifera at the colony level, even when some individuals are lost. However, ingestion leads to high mortality. The reported sublethal effects are highly decreased longevity, a reduction in $\mathrm{pH}$-values in the digestive system and the hemolymph, and an increased responsiveness to water. The shift towards stronger acidity after treatment confirms that damage to the epithelial tissue and organs is likely to be caused by hyperacidity. Pathological repercussions e.g., degeneration of rectal epithelium, malpighian tubules and ventriculus may also occur.

These results indicate a general impairment of the bees after treatment. The treatment in autumn or winter affects primarily long-living winter bees which are essential for winter survival and successful colony development in the spring. Treatment during summer with brood can cause substantial brood damage. Even when treating artificial swarms or nucleus colonies it cannot be certain that damages will not occur due to the extensive exposure to OAD in the colony. Long-term effects such as reduced amount of brood in treated colonies have been reported.

OAD is one of the most important organic acids used for the control of $V$. destructor. It is indispensable but must be dosed precisely and applied as seldom as possible to prevent sublethal damages which eventually lead to the loss of bees. Long disposition in the bee hive can cause accumulation of the acid and therefore induce further damage.

Acknowledgments: We would like to thank the Tierärztliche Klinik für Pferde Seeburg, Germany for their cooperation.

Author Contributions: Eva Rademacher, Marika Harz and Saskia Schneider conceived and designed the experiments; Marika Harz and Saskia Schneider performed the experiments; Eva Rademacher, Marika Harz and Saskia Schneider analyzed the data; Eva Rademacher, Marika Harz and Saskia Schneider wrote the paper. 
Conflicts of Interest: The authors declare no conflict of interest.

\section{References}

1. Popov, E.T.; Melnik, V.N.; Matchinev, A.N. Application of oxalic acid in varroatosis. In XXXII International Congress Apimondia; Apimondia Publication: Bucharest, Romania, 1989; p. 149.

2. Nanetti, A.; Büchler, R.; Charriere, J.D.; Fries, I.; Helland, S.; Imdorf, A.; Korpela, S.; Kristiansen, P. Oxalic acid treatments for varroa control (review). Apiacta 2003, 38, 81-87.

3. Rademacher, E.; Imdorf, A. Legalization of the use of oxalic acid in Varroa control. Bee World 2004, 85, 70-72. [CrossRef]

4. Rademacher, E.; Harz, M. Oxalic acid for the control of Varroosis in honey bee colonies-A review. Apidologie 2006, 37, 98-120. [CrossRef]

5. Rademacher, E. Oxalsäure als Tierarzneimittel zur Bekämpfung der Varroose legal einsetzbar. ADIZ/die biene/Imkerfreund 2006, 2, 9. (In German)

6. Dade, H.A. Anatomy and Dissection of the Honeybee; Bee Research Association Publications: London, UK, 1977.

7. Greco, M.K. Imaging techniques for improved bee management. ALP Sci. 2010, 534, 1-38.

8. Rademacher, E.; Fahlberg, A.; Raddatz, M.; Schneider, S.; Voigt, K. Galenics: Studies of the toxicity and distribution of sugar substitutes on Apis mellifera. Apidologie 2013, 44, 222-233. [CrossRef]

9. Kanao, T.; Okamoto, T.; Miyachi, Y.; Nohara, N. Parental exposure to low dose X-rays in Drosophila melanogaster includes early emergence in offspring, which can be modulated by transplantation of polar cytoplasm. Mutat. Res. 2003, 527, 1-6. [CrossRef]

10. Rademacher, E.; Harz, M. Oxalic acid: Toxicology on Apis mellifera. Apidologie 2009, 40, 651-669.

11. Aliano, N.P.; Ellis, M.D.; Siegfried, B.D. Acute contact toxicity of oxalic acid to Varroa destructor (Acari: Varroidae) and their Apis mellifera (Hymenoptera: Apidae) hosts in laboratory bioassays. J. Econ. Entomol. 2006, 99, 1579-1582. [CrossRef] [PubMed]

12. Carrasco-Letelier, L.; Mendoza, Y.; Ramallo, G. Acute contact toxicity of oxalic acid on honeybees in the southwestern zone of Uruguay. Chil. J. Agric. Res. 2012, 72, 285-289. [CrossRef]

13. Desneux, N.; Decourtye, A.; Delpuch, J.M. The sublethal effects of pesticides on beneficial arthropods. Annu. Rev. Entomol. 2007, 52, 81-106. [CrossRef] [PubMed]

14. Rouibi, A.; Bouchema, W.; Loucif-Ayad, W.; Achou, M.; Soltani, N. Risks assessment of two acaricides (fluvalinate ad oalic acid) in Apis mellifera intermissa (Hymenoptera, Apidae): Acethylcholinestease and glutathione S-trasferase activities. J. Entomol. Zool. Stud. 2016, 4, 503-508.

15. Brosgaard, C.J.; Jensen, S.E.; Hansen, C.W.; Hansen, H. Spring treatment with oxalic acid in honeybee colonies as Varroa control. DIAS Rep. Hortic. 1999, 6, 1-16.

16. Gregorc, A.; Pogaènik, A.; Bowen, I.D. Cell death in honeybee (Apis mellifera) larvae treated with oxalic acid. Apidologie 2004, 35, 453-460. [CrossRef]

17. Hatjina, F.; Haristos, L. Indirect effects of oxalic acid administration by trickling method on bee brood. J. Apicult. Res. 2005, 44, 172-174. [CrossRef]

18. Moosbeckhofer, R.; (AGES, Vienna, Austria); Rademacher, E.; (Freie Universität Berlin, Berlin, Germany). Personal communication, 2012.

19. Higes, M.; Meana, A.; Suarez, M.; Llorente, J. Negative long-term effects on bee colonies treated with oxalic acid against Varroa jacobsoni Oud. Apidologie 1999, 30, 289-292. [CrossRef]

20. Martin-Hernandez, R.; Higes, M.; Perez, J.L.; Nozal, M.J.; Gomez, L.; Meana, A. Short term negative effect of oxalic acid in Apis mellifera iberiensis. Span. J. Agric. Res. 2007, 5, 474-480. [CrossRef]

21. Nozal, M.J.; Bernal, J.; Gomez, L.; Higes, M.; Meana, A. Determination of oxalic acid and other organic acids in honey and in some anatomic structures of bees. Apidologie 2003, 34, 181-188. [CrossRef]

22. Nanetti, A.; Ghini, S.; Gattavecchia, E.; Bartolomei, P.; Marcazzan, G.L.; Massi, S. Pharmacodynamics of Oxalic Acid and Treatment Residues in Honey; European Group for Integrated Varroa Control (CA 3686): Rauischholzhausen, Germany, 2003.

23. Raddatz, M.; Rademacher, E. Sublethal effects of oxalic acid on Apis mellifera L. (Hymenoptera: Apidae): Pharmacodynamics. Apidologie 2010, 41, 676-694.

24. Schneider, S.; Eisenhardt, D.; Rademacher, E. Sublethal effects of oxalic acid on Apis mellifera L. (Hymenoptera Apidae): Changes in behavior and longevity. Apidologie 2012, 43, 218-225. [CrossRef] 
25. O'Neil, M.J. The Merck Index: An Encyclopedia of Chemicals, Drugs and Biologicals, 13th ed.; Whitehouse Station: New York, NY, USA, 2001.

26. Charriere, J.D.; Imdorf, A. Oxalic acid treatment by trickling against Varroa destructor: Recommendations for use in Central Europe and under temperate climate conditions. Bee World 2002, 83, 51-60. [CrossRef]

27. Toomemaa, K.; Martin, A.; Williams, I. The effect of different concentrations of oxalic acid in aquaeous and sucrose solution on Varroa mites and honey bees. Apidologie 2010, 41, 634-653. [CrossRef]

28. Aliano, N.P.; Ellis, M.D. Bee-to-bee contact drives oxalic acid distribution in honey bee colonies. Apidologie 2008, 39, 481-487. [CrossRef]

29. Rademacher, E.; Harz, M.; Schneider, S. The development of HopGuard ${ }^{\circledR}$ as a winter treatment against Varroa destructor in colonies of Apis mellifera. Apidologie 2015, 46, 748-759. [CrossRef]

(C) 2017 by the authors. Licensee MDPI, Basel, Switzerland. This article is an open access article distributed under the terms and conditions of the Creative Commons Attribution (CC BY) license (http:/ / creativecommons.org/licenses/by/4.0/). 